\title{
Changing the Landscape of the HIV Epidemic among MSM in China: Results from Three Consecutive Respondent-Driven Sampling Surveys from 2009 to 2011
}

\author{
Xuefeng Li, ${ }^{1,2}$ Hongyan Lu, ${ }^{3}$ Catherine Cox, ${ }^{4}$ Yuejuan Zhao, ${ }^{3}$ Dongyan Xia, ${ }^{3}$ Yanming Sun, \\ Xiong He, ${ }^{3}$ Yan Xiao, ${ }^{1}$ Yuhua Ruan, ${ }^{1}$ Yujiang Jia, ${ }^{5}$ and Yiming Shao ${ }^{1}$ \\ ${ }^{1}$ State Key Laboratory for Infectious Disease Prevention and Control, National Center for AIDS/STD Control and Prevention, \\ Chinese Center for Disease Control and Prevention, Collaborative Innovation Center for Diagnosis and \\ Treatment of Infectious Diseases, No. 155 Changbai Road, Changping District, Beijing 102206, China \\ ${ }^{2}$ Karamay Center for Disease Control and Prevention, Karamay 834000, China \\ ${ }^{3}$ Beijing Center for Disease Control and Prevention, Beijing 100013, China \\ ${ }^{4}$ School of Public Health, University of Maryland, College Park, MD 20742, USA \\ ${ }^{5}$ Department of Preventive Medicine, Vanderbilt University School of Medicine, Nashville, TN 37232, USA
}

Correspondence should be addressed to Yiming Shao; yshao08@gmail.com

Received 12 September 2013; Accepted 27 December 2013; Published 20 January 2014

Academic Editor: Jennifer Huang Bouey

Copyright (C) 2014 Xuefeng Li et al. This is an open access article distributed under the Creative Commons Attribution License, which permits unrestricted use, distribution, and reproduction in any medium, provided the original work is properly cited.

\begin{abstract}
This study assessed the changes of HIV incidence and its predictors among Beijing's men who have sex with men (MSM). Three consecutive cross-sectional surveys were carried out using a consistent respondent-driven sampling (RDS) approach in 2009, 2010, and 2011, respectively. Structured-questionnaire based interviews were completed with computer-assisted self-administration. Incident infection was examined with BED capture enzyme immunoassay (BED-CEIA). The overall rate of HIV prevalence was $8.0 \%$ in the three years (95\% confidence interval [CI]: 4.9\%-11.2\%). The overall rate of BED-CEIA incidence was 7.8/100 person years (PY) (95\% CI: 5.5-10.1) with 6.8/100PY (95\% CI: 3.4-10.2) in 2009, 11.2/100PY (95\% CI: 6.2-16.3) in 2010, and 5.8/100PY (95\% CI: 2.4-9.3) in 2011, respectively. Multivariable logistic regression analysis revealed that, compared with HIV-negative MSM, recently infected MSM were more likely to be bisexual (adjusted odds ratio [AOR] $=2.1,95 \%$ CI: 1.1-4.1), live in Beijing $\leq 3$ years $(\mathrm{AOR}=2.1,95 \% \mathrm{CI}: 1.2-4.0)$, and have a negative attitude towards safe sex (AOR = 1.1 per scale point, 95\% CI: 1.0-1.1). This study demonstrated a disturbing rise of HIV infections among Beijing's MSM. These findings underscored the urgency of scaling up effective and better-targeted intervention services to stop the rapid spread of the virus.
\end{abstract}

\section{Introduction}

Historically, the HIV epidemic in China has been confined to certain high-risk populations such as injection drug users (IDUs) and former blood and plasma donors in geographically disparate rural areas [1-6]. In recent years, the synthesized data suggested that HIV transmission has begun to shift from IDUs to populations at risk through unprotected sex, either through heterosexual contacts or male homosexual sex, accounting for nearly half of all new infections in 2007 [7]. Prevalence of HIV and other sexually transmitted diseases (STDs), for example, syphilis, is rapidly increasing among MSM in major cities [8-17]. MSM have emerged as a high-risk group for HIV in China. A metaanalysis revealed that the overall HIV prevalence among MSM in China increased substantially from $1.4 \%$ during 2001-2003 to $2.3 \%$ during $2004-2006$ and to $5.3 \%$ during 2007-2009 [18]. The sentinel surveillance demonstrated that the overall HIV prevalence among MSM increased from 0.9\% in 2003 to $6.3 \%$ in 2011 [19].

HIV prevalence is one of the key indicators to describe the disease burden. However, using the prevalence alone is 
difficult to capture the dynamic changes of the HIV epidemic, especially in the context of the expansion of antiretroviral treatment along with a spectrum of preventive intervention activities combined with a variety of behavioral, social, and structural factors that affected the spread of the virus. The emerging epidemic among MSM underscores the urgent need for the incidence measurement. Monitoring HIV incidence would pinpoint current dynamics of HIV infection and optimize the resource allocation for prevention activities that would result in the most HIV infections averted, resulting in the lowest HIV transmission rate possible.

There are three main ways to calculate HIV incidence. The "gold standard" method is a prospective cohort study; however, its disadvantages are it is expensive, difficult to maintain retention rate, lack of representative sampling, and the Hawthorne effect which affects a subject's behavior and may produce a bias conclusion $[20,21]$. The second method is the mathematical model based on HIV prevalence data, but that relies on locally appropriate mortality data and survival time distribution [22]. Comparatively, laboratorybased methods for incidence estimation in a single crosssectional survey have more reliability and attraction [20]. One commercialized and widely used assay to detect and distinguish recent from long-term infections is BED capture enzyme immunoassay (BED-CEIA), which has been applied to different populations worldwide [23-26]. In this study, we assessed the rates of recent HIV infections and its correlates using the BED-CEIA among MSM.

\section{Methods}

2.1. Study Design, Recruitment, and Instrument. Three consecutive cross-sectional RDS surveys were conducted among Beijing MSM from September to October 2009, from October 2010 to early January 2011, and from September to December 2011, respectively. The criteria for recruiting participants included men who had a valid recruitment coupon or who were selected as a seed, had engaged in homosexual behavior in the past 12 months (sex was defined as oral, anal, or mutual masturbation), currently living or working in Beijing, were at least 18 years old, and were able to provide written, informed consent. Structured questionnaire based interviews were conducted in an HIV voluntary counseling and testing clinic (VCT) located at the Beijing Center for Disease Control and Prevention.

Respondent-driven sampling (RDS) was used to recruit participants for each cycle, of which the details of the sampling method have been previously described [27]. Briefly, each recruitment chain started with 7-10 selected "seeds" that were given three coupons to recruit up to 3 eligible peers from their social network, who in turn were screened, enrolled, interviewed, were asked to refer 3 participants, and so on until both sample size and equilibrium were reached. Seeds were chosen from diverse networks with respect to geography (e.g., bar, bathhouse, park, and internet), demographic characteristics, and subculture, and they should be sociometric stars, articulate, and motivated. There are 7, 10, and 8 seeds in 2009, 2010, and 2011. Repeated seeds were 3, 3, and 2 between 2009 and 2010, between 2009 and 2011, and between 2010 and 2011, respectively. After signing the informed consent, we used computer-assisted self-administration to collect information on demographic characteristics, HIV risk behaviors, HIV testing history, drug use, access to HIV-related prevention services, and attitudes and perceptions of abilities for safe sex, where the psychosocial scale has been described previously [28]. This was assessed by a 15-item scale asking participants' self-efficacy in having safe sex (strongly disagree; somewhat disagree; somewhat agree; strongly agree), where the increasing scale score means greater negative attitudes and perceptions of abilities towards safe sex, and the internal reliability was high with a Cronbach's alpha value of 0.94 in our study. Being a bisexual was defined as someone who was married and/or self-reported to ever have had sex with a woman.

At the completion of the interview, a blood sample was obtained to test for HIV and syphilis. Participants were given $30 \mathrm{RMB}$ for completion of the survey and $20 \mathrm{RMB}$ for recruitment of their peers as monetary incentive. Pre- and postcounseling were offered to all participants and testing results were given within 1 to 3 weeks. At the time of specimen collection, the participant was given an appointment card with the name, telephone number, and email of the counselor, as well as the date, time, and location of their appointment for results counseling. Alternatively, the participant can arrange to contact the trained research staff for their results by telephone. BED results were not provided for participants because the main function of BED assay is to estimate population incidence rather than diagnose recent infection for an individual [20]. Those who were tested positive for HIV or syphilis were referred to appropriate medical and mental health and social support services as needed.

2.2. Laboratory Methods. Blood samples were tested for HIV and syphilis antibodies. HIV enzyme immunosorbent assay (Vironostika HIV Uni-Form plus O, bioMerieux, The Netherlands) was used for HIV screening with a Western blot for confirmation (HIV Blot 2.2 WBTM, Genelabs Diagnostics, Singapore). Syphilis infection was determined using a rapid plasma reagin (RPR) test (Shanghai Rongsheng, China) and confirmed by the Treponema pallidum particle assay (TPPA) test (Fujirebio Inc., Japan). The BED IgG-capture ELISA (BED-CEIA, Calypte Biomedical Corporation, Rockville, Maryland) was applied to HIV-positive specimens for the detection of recent HIV seroconversions and estimation of HIV incidence. The basis of the assay is that it measures the ratio of HIV-specific IgG to total IgG, where the proportion slowly rises following seroconversion providing an indicator of disease progression [29]. Samples that had a normalized optical density (OD) less than or equal to the cutoff of 0.8 on the BED assay were classified as recent infections (seroconversion occurring $\leq 153$ days), and others were considered as long-term infections. The calculation of HIV incidence followed US CDC's recommended formulas.

2.3. Statistical Analyses. Statistical analysis was performed using SAS version 9.1, where results with a $P<0.05$ were considered significant. Of note, MSM who participated in the survey repeatedly were excluded from analysis in order 
to estimate the trends of HIV infection accurately. Chisquared test was applied to compare sample demographic and behavior characteristics across survey years. To explore risk factors associated with recent HIV infection, we compared sociodemographic characteristics and behaviors of recently infected MSM to those of HIV-negative MSM using univariate logistic regression and forward stepwise multivariate logistic regression. Variables that were statistically significant in univariate analysis $(P<0.05)$ were entered into multivariate analysis. Considering the current limitations of RDSAT for conducting regression analyses [30] and the fact that the weighted RDS was performed for three individual years and no different results were found compared with the unweighted analytical method, it was determined that the time gap would have an effect on the sampling population and therefore we did not use weighted RDS analysis for the combined three cycles of data.

The study protocol and informed consent forms were reviewed and approved by the Institutional Review Boards of the National Center for AIDS/STD Control and Prevention (NCAIDS) in China, Vanderbilt University, and the University of California, San Francisco.

\section{Results}

3.1. Characteristics of Participants. A total of 501, 501, and 502 subjects were recruited in 2009, 2010, and 2011, respectively. Of the 1504 MSM assessed and screened in the three consecutive surveys, 4 participants were excluded due to being younger than 18 years old (1 participant in 2009), reporting no sexual behavior in the past year (1 participant in 2010), and having no valid recruitment card (2 participants in 2011). A total of 1500 eligible participants completed the questionnaire and provided blood samples. In the second cycle, 115 MSM participated in the first cycle repeatedly, and, in the third cycle, 57 participated in the first cycle and 28 participated in the second cycle (12 MSM participated in both the first and second cycle). As noted previously, 188 repeated participants were removed from the final sample for analysis.

Demographic and behavioral characteristics of study participants for each cycle are presented in Table 1. Major variables are different across the 3 cycles without a clear trend. In the subjects, more college or higher education level, employed, and urban MSM were recruited across the survey years. The proportion of MSM who were aware of the HIV status of the most recent male partner increased over the years from $26.4 \%$ in 2009 to $37.5 \%$ in 2011 , and the proportion reporting knowing where to get an HIV test also rose from $94.2 \%$ in 2009 to $100.0 \%$ in 2011 . The proportion reporting having a female sexual partner in the past 6 months decreased from $20.8 \%$ in 2009 to $10.8 \%$ in 2011 , whereas the rates of unprotected sex with male or female partners both remained stably high.

3.2. HIV/Syphilis Prevalence and BED Estimated Incidence. Among 1312 participants, it was determined that 104 were infected with HIV and 1208 were HIV-negative. According to BED results, of the $104 \mathrm{HIV}$ positive MSM, 45 were categorized as recently infected and 59 were long-term infections. The ratio of incidence infection to overall infection for 3 survey years was $41.7 \%$ (15/36), 50\% (19/38), and $36.7 \%$ (11/30), respectively. The overall rate of BED-CEIA incidence was 7.8/100 person years (PY) (95\% confidence interval (CI): 5.5-10.1) with 6.8/100 PY (95\% CI: 3.4-10.2) in 2009, 11.2/100 PY (95\% CI: 6.2-16.3) in 2010, and 5.8/100 PY (95\% CI: 2.4-9.3) in 2011, respectively. Overall, HIV prevalence rose from $7.2 \%$ in 2009 to $9.9 \%$ in 2010 and dropped to $7.0 \%$ again in 2011 yet remaining at a high level, while, at the same time, syphilis prevalence decreased from $22.0 \%$ in 2009 to $12.2 \%$ in 2010 and $10.5 \%$ in 2011.

3.3. Factors Associated with Recent HIV Infection. Table 2 presents the univariate and multivariate logistic analyses of variables associated with recent HIV infection among Beijing MSM. Multivariate logistic regression analyses indicated that compared with HIV-negative MSM, recently infected MSM were significantly more likely to be bisexual (adjusted odds ratio $(\mathrm{AOR})=2.1,95 \% \mathrm{CI}: 1.1-4.1)$, live in Beijing $\leq 3$ years $(\mathrm{AOR}=2.1,95 \% \mathrm{CI}: 1.2-4.0)$, and have a negative attitude towards safe sex $(\mathrm{AOR}=1.1$ per scale point, 95\% CI: $1.0-1.1)$.

\section{Discussion}

This study revealed the alarmingly high HIV prevalence and incidence rates among Beijing MSM in the past three years. The prevalence is higher than the rates found by previous RDS surveys among Beijing MSM (0.4\% in 2004, 4.6\% in 2005, and $5.8 \%$ in 2006) [31]. The BED-CEIA estimated incidence is much higher than previous rates estimated in either crosssectional studies in 2005-06 (2.9/PY in 2005, 3.6/PY in 2006) [32] or cohort study in 2007 (2.6/PY) in the same population in Beijing [16], but the rates found in this study are similar to those reported from several cities, such as $7.01 \%, 7.98 \%$, and $7.8 \%$ for Chongqing from 2006 to 2008 [33], 15.43\% in 2009 [34], and 7.54\% for Jiangsu in 2008 [35]. MSM as a high-risk population in China are facing a severe challenge against HIV infection, and the synthesized data revealed a disturbing expansion in the epidemic among MSM in China. The findings from these three consecutive surveys signals the beginning in changing the landscape of the HIV epidemic in China. HIV transmission has begun to shift from the historically main transmission route, IDU, to populations at risk through unprotected heterosexual contacts or male homosexual sex. The unprotected sex between men has been emerging as a major route of HIV transmission in China like many other nations surrounding China which experienced a resurgence in HIV transmission among MSM [36, 37].

In this study, HIV prevalence and BED-CEIA incidence estimates were very close, and the ratio of incidence infection to overall infection for 3 survey years was between $40 \%$ and $50 \%$, which might demonstrate that the recent infection contributes to the main cause of the expanding HIV epidemic; therefore, it is important to identify the characteristics of recently infected MSM.

This study found that recently infected MSM were significantly more likely to be bisexual, and this finding is consistent with the conclusion from a recent meta-analysis, which indicated that bisexual behavior was significantly associated 
TABLE 1: Demographic and behavior characteristics of MSM, Beijing, China, 2009-2011.

\begin{tabular}{|c|c|c|c|c|c|c|c|}
\hline \multirow{2}{*}{ Variable } & \multicolumn{2}{|c|}{$2009(n=500)$} & \multicolumn{2}{|c|}{$2010(n=385)$} & \multicolumn{2}{|c|}{$2011(n=427)$} & \multirow{2}{*}{$P$} \\
\hline & $N$ & $\%$ & $N$ & $\%$ & $N$ & $\%$ & \\
\hline \multicolumn{8}{|l|}{ Age group in years } \\
\hline $18-25$ & 173 & 34.6 & 154 & 40.0 & 147 & 34.4 & \multirow{3}{*}{0.5934} \\
\hline $26-35$ & 205 & 41.0 & 156 & 40.5 & 186 & 43.6 & \\
\hline $36-71$ & 122 & 24.4 & 75 & 19.5 & 94 & 22.0 & \\
\hline Education, completed college or more & 174 & 34.8 & 172 & 44.7 & 208 & 48.7 & $<0.0001$ \\
\hline Married to a woman currently & 104 & 20.8 & 56 & 14.6 & 68 & 15.9 & 0.0325 \\
\hline Living with boyfriend & 104 & 20.8 & 74 & 19.2 & 101 & 23.7 & 0.2896 \\
\hline \multicolumn{8}{|l|}{ Employment status } \\
\hline Employed & 454 & 90.8 & 365 & 94.8 & 416 & 97.4 & \multirow{2}{*}{$<0.0001$} \\
\hline Unemployed & 46 & 9.2 & 20 & 5.2 & 11 & 2.6 & \\
\hline \multicolumn{8}{|l|}{ Residence } \\
\hline Urban & 396 & 79.2 & 343 & 89.1 & 389 & 91.5 & \multirow{2}{*}{$<0.0001$} \\
\hline Rural & 104 & 20.8 & 42 & 10.9 & 36 & 8.5 & \\
\hline Living three years or fewer in Beijing & 221 & 44.2 & 168 & 43.6 & 184 & 43.1 & 0.9440 \\
\hline \multicolumn{8}{|l|}{ Mean monthly income (RMB), P12M } \\
\hline None & 58 & 11.6 & 41 & 10.7 & 30 & 7.0 & \multirow{5}{*}{$<0.0001$} \\
\hline$<1000$ & 47 & 9.4 & 19 & 4.9 & 11 & 2.6 & \\
\hline $1000-2999$ & 271 & 54.2 & 173 & 44.9 & 144 & 33.7 & \\
\hline $3000-4999$ & 80 & 16.0 & 64 & 16.6 & 137 & 32.1 & \\
\hline$\geq 5000$ & 44 & 8.8 & 88 & 22.9 & 105 & 24.6 & \\
\hline Have health insurance & 236 & 47.2 & 209 & 54.3 & 229 & 53.6 & 0.0590 \\
\hline \multicolumn{8}{|l|}{ Self-reported sexual orientation } \\
\hline Homosexual & 346 & 69.2 & 278 & 72.2 & 313 & 73.3 & \multirow{4}{*}{0.0693} \\
\hline Heterosexual & 5 & 1.0 & 7 & 1.8 & 2 & 0.5 & \\
\hline Bisexual & 147 & 29.4 & 93 & 24.2 & 109 & 25.5 & \\
\hline Do not know & 2 & 0.4 & 7 & 1.8 & 3 & 0.7 & \\
\hline \multicolumn{8}{|l|}{ Self-reported preferred role in anal sex with men } \\
\hline Equally & 291 & 59.4 & 235 & 63.3 & 234 & 56.0 & \multirow{3}{*}{0.3509} \\
\hline Insertive & 133 & 27.1 & 91 & 24.5 & 124 & 29.7 & \\
\hline Receptive & 66 & 13.5 & 45 & 12.1 & 60 & 14.4 & \\
\hline Had male sexual partner, P6M & 488 & 97.6 & 373 & 96.9 & 427 & 100.0 & 0.0020 \\
\hline More than five male sex partners, P6M & 144 & 28.8 & 55 & 14.3 & 73 & 17.1 & $<0.0001$ \\
\hline \multicolumn{8}{|l|}{ Type of most recent male partner, $\mathrm{P} 6 \mathrm{M}$} \\
\hline Regular & 309 & 63.3 & 205 & 55.0 & 267 & 62.5 & \multirow{2}{*}{0.0281} \\
\hline Casual & 179 & 36.7 & 168 & 45.0 & 160 & 37.5 & \\
\hline \multicolumn{8}{|l|}{ Venues of founding/meeting last male partner, P6M } \\
\hline Internet/bar/massage/club & 306 & 63.1 & 274 & 73.5 & 300 & 70.3 & \multirow{2}{*}{0.0033} \\
\hline Bathhouse/sauna/park/public rest room & 179 & 36.9 & 99 & 26.5 & 127 & 29.7 & \\
\hline \multicolumn{8}{|l|}{ Venues of sex with last male partner, P6M } \\
\hline Residence/bar/club/hotel & 428 & 87.7 & 341 & 91.4 & 370 & 86.7 & 0.0894 \\
\hline Bathhouse/sauna/park/public rest room & 60 & 12.3 & 32 & 8.6 & 57 & 13.4 & 0.0074 \\
\hline $\begin{array}{l}\text { Unprotected insertive anal sex with last male partner, } \\
\text { P6M }\end{array}$ & 127 & 36.6 & 93 & 38.9 & 112 & 40.4 & 0.6118 \\
\hline $\begin{array}{l}\text { Unprotected receptive anal sex with last male partner, } \\
\mathrm{P} 6 \mathrm{M}\end{array}$ & 115 & 40.4 & 99 & 50.0 & 95 & 42.8 & 0.1026 \\
\hline Knew the HIV status of most recent male partner & 129 & 26.4 & 133 & 35.7 & 160 & 37.5 & 0.0007 \\
\hline Disclosed HIV status to most recent male partner & 236 & 48.4 & 180 & 48.3 & 230 & 53.9 & 0.1727 \\
\hline
\end{tabular}


TABLE 1: Continued.

\begin{tabular}{|c|c|c|c|c|c|c|c|}
\hline \multirow{2}{*}{ Variable } & \multicolumn{2}{|c|}{$2009(n=500)$} & \multicolumn{2}{|c|}{$2010(n=385)$} & \multicolumn{2}{|c|}{$2011(n=427)$} & \multirow{2}{*}{$P$} \\
\hline & $N$ & $\%$ & $N$ & $\%$ & $N$ & $\%$ & \\
\hline Bisexual & 104 & 20.8 & 55 & 14.3 & 66 & 15.5 & 0.0206 \\
\hline Ever had sex with a woman & 283 & 56.6 & 181 & 47.0 & 207 & 48.5 & 0.0075 \\
\hline Had female sexual partner, P6M & 104 & 20.8 & 49 & 12.7 & 46 & 10.8 & $<0.0001$ \\
\hline Unprotected sex with most recent female partner, P6M & 75 & 72.1 & 41 & 85.4 & 35 & 76.1 & 0.2024 \\
\hline Knows where to get HIV test & 470 & 94.2 & 383 & 99.5 & 427 & 100.0 & $<0.0001$ \\
\hline \multicolumn{8}{|l|}{ HIV testing history } \\
\hline Never tested & 166 & 33.2 & 148 & 38.4 & 93 & 21.8 & \multirow{5}{*}{$<0.0001$} \\
\hline Tested more than one year prior to interview & 68 & 13.6 & 48 & 12.5 & 49 & 11.5 & \\
\hline Tested within the past year & 55 & 11.0 & 55 & 14.3 & 49 & 11.5 & \\
\hline Tested within the past 6 months & 142 & 28.4 & 102 & 26.5 & 197 & 46.1 & \\
\hline Tested within the past 3 months & 69 & 13.8 & 32 & 8.3 & 39 & 9.1 & \\
\hline Received free condoms/lubricant, $\mathrm{P} 12 \mathrm{M}$ & 436 & 87.2 & 267 & 69.4 & 330 & 77.3 & $<0.0001$ \\
\hline Bought condoms, P12M & 249 & 49.8 & 234 & 60.8 & 246 & 57.6 & 0.0029 \\
\hline Drug use, $\mathrm{P} 12 \mathrm{M}$ & 4 & 0.8 & 2 & 0.5 & 4 & 0.9 & 0.7847 \\
\hline Smoking, P12M & 217 & 43.4 & 154 & 40.0 & 153 & 35.8 & 0.0640 \\
\hline \multicolumn{8}{|l|}{$\begin{array}{l}\text { Level of HIV risk via homosexual contacts for } \\
\text { self-perception }\end{array}$} \\
\hline Great & 63 & 12.6 & 28 & 7.3 & 42 & 9.8 & \multirow{4}{*}{0.4414} \\
\hline Moderate & 92 & 18.4 & 80 & 20.8 & 75 & 17.6 & \\
\hline Small & 286 & 57.2 & 202 & 52.6 & 272 & 63.7 & \\
\hline None & 59 & 11.8 & 74 & 19.3 & 38 & 8.9 & \\
\hline Current test HIV positive & 36 & 7.2 & 38 & 9.9 & 30 & 7.0 & 0.2433 \\
\hline Current test syphilis positive & 110 & 22.0 & 47 & 12.2 & 45 & 10.5 & $<0.0001$ \\
\hline
\end{tabular}

${ }^{*}$ RMB: renminbi, approximately US \$0.15; P12M: in the past 12 months; P6M: in the past 6 months; chi-squared test was used to compare proportions.

with a $30 \%$ increased risk of HIV infection [38]. In the present study, the three-year overall percentage of bisexual MSM and currently married MSM was $17.1 \%$ and $17.4 \%$, respectively. The marriage rate was in agreement with the result from a systematic review which reported a national average of $17.0 \%$, higher than most Western developed countries [39]. Chinese MSM suffer from both HIV-related and homosexualityrelated stigma [40,41], where they get married to cover their homosexual orientation, which when combined, these married MSM can be difficult to be reached and intervened. Common bisexual behaviors combined with inconsistent condom use put themselves and their female partner at high risk for HIV exposure. Further research is needed to understand the bridging effect of transmitting HIV from MSM to their female partners.

This study also found that MSM living in Beijing $\leq 3$ years compose a risk group of recent HIV infection. Compared with longtime residents, recent migrants may lack HIV-related knowledge, have a low level of HIV infection awareness, and lack information about HIV/AIDS-related healthcare services, such as voluntary counseling and testing, condom distribution programs, and free antiretroviral therapy. Recent migrants are at particular risk for HIV infection; therefore, it is important to improve their access to HIV prevention and treatment services, and further study is needed to explore the differences between longtime and short-term residents.

Previously published studies among Chinese or foreign MSM indicated that unprotected anal sex was a predictor of recent HIV infection [42, 43], and, although we did not find that relationship in our study, we found that holding a negative attitude towards safe sex practices was associated with recent HIV infection. In the three annual cross-sectional surveys, more than two-thirds of MSM had multiple male sex partners in the past 6 months, and about two-fifths reported unprotected insertive or receptive anal sex in the past 6 months over the period. Given the common multiple sexual partnerships and low rate of condom use in this population, more health education and harm reduction programs are urgently needed to change their low perception of HIV infection risk and increase their confidence in practicing safer sex.

We recognized the limitations of the study. First, the questionnaire involves private and sensitive HIV risk behaviors, so participants may choose to give socially desirable answers instead of true effects, which might introduce information bias. Second, the cross-sectional study design cannot establish the causality between associated factors and recent HIV infection. Third, due to misclassification of long-term HIV infection as recent infection among persons who are on 
TABLE 2: Risk factors associated with recent HIV infection among MSM, 2009-2011 (recent HIV infection versus HIV negative, $N=1253$ ).

\begin{tabular}{|c|c|c|c|c|c|}
\hline Variable & Recent infection \% $(N)$ & OR $(95 \% \mathrm{CI})$ & $P$ & $\operatorname{AOR}(95 \% \mathrm{CI})$ & $P$ \\
\hline \multicolumn{6}{|l|}{ Age group in years } \\
\hline $18-25$ & $3.5(16)$ & 1.0 & & & \\
\hline $26-35$ & $3.3(17)$ & $0.9(0.5-1.9)$ & 0.8227 & & \\
\hline $36-71$ & $4.4(12)$ & $1.2(0.6-2.7)$ & 0.5706 & & \\
\hline \multicolumn{6}{|l|}{ Education } \\
\hline College or above & $3.1(17)$ & 1.0 & & & \\
\hline High school or less & $3.9(28)$ & $1.3(0.7-2.3)$ & 0.4575 & & \\
\hline \multicolumn{6}{|l|}{ Current marital status } \\
\hline Unmarried & $3.1(32)$ & 1.0 & & & \\
\hline Married & $6.0(13)$ & $2.0(1.0-3.9)$ & 0.0386 & & \\
\hline \multicolumn{6}{|l|}{ Living with boyfriend } \\
\hline Yes & $1.5(4)$ & 1.0 & & & \\
\hline No & $4.2(41)$ & $2.9(1.0-8.1)$ & 0.0453 & & \\
\hline \multicolumn{6}{|l|}{ Employment status } \\
\hline Employed & $3.7(44)$ & 1.0 & & & \\
\hline Unemployed & $1.5(1)$ & $0.4(0.1-2.9)$ & 0.3600 & & \\
\hline \multicolumn{6}{|l|}{ Residence } \\
\hline Urban & $3.6(39)$ & 1.0 & & & \\
\hline Rural & $3.5(6)$ & $1.0(0.4-2.3)$ & 0.9219 & & \\
\hline \multicolumn{6}{|l|}{ Years living in Beijing } \\
\hline$>3$ & $2.5(18)$ & 1.0 & & 1.0 & \\
\hline$\leq 3$ & $5.0(27)$ & $2.0(1.1-3.7)$ & 0.0221 & $2.1(1.2-4.0)$ & 0.0153 \\
\hline \multicolumn{6}{|l|}{ Mean monthly income (RMB), P12M } \\
\hline$\geq 1000$ & $3.6(38)$ & 1.0 & & & \\
\hline$<1000$ & $3.7(7)$ & $1.0(0.5-2.3)$ & 0.9523 & & \\
\hline \multicolumn{6}{|l|}{ Have health insurance } \\
\hline Yes & $3.2(21)$ & 1.0 & & & \\
\hline No & $4.0(24)$ & $1.2(0.7-2.3)$ & 0.4771 & & \\
\hline \multicolumn{6}{|l|}{ Self-reported sexual orientation } \\
\hline Others & $4.1(15)$ & 1.0 & & & \\
\hline Homosexual & $3.4(30)$ & $0.8(0.4-1.5)$ & 0.5443 & & \\
\hline \multicolumn{6}{|c|}{ Self-reported preferred role in anal sex with men } \\
\hline Equally & $4.6(33)$ & 1.0 & & & \\
\hline Insertive & $2.1(7)$ & $0.4(0.2-1.0)$ & 0.0487 & & \\
\hline Receptive & $3.1(5)$ & $0.7(0.3-1.7)$ & 0.3875 & & \\
\hline \multicolumn{6}{|l|}{ No. of male sex partners, P6M } \\
\hline$\leq 5$ & $3.0(30)$ & 1.0 & & & \\
\hline$>5$ & $5.9(15)$ & $2.0(1.1-3.8)$ & 0.0318 & & \\
\hline \multicolumn{6}{|l|}{ Type of most recent male partner, P6M } \\
\hline Regular & $3.3(25)$ & 1.0 & & & \\
\hline Casual & $4.2(20)$ & $1.3(0.7-2.3)$ & 0.4473 & & \\
\hline \multicolumn{6}{|c|}{ Venues of founding/meeting last male partner, P6M } \\
\hline Internet/bar/massage/club & $3.9(33)$ & 1.0 & & & \\
\hline Bathhouse/sauna/park/public rest room & $3.2(12)$ & $0.8(0.4-1.6)$ & 0.5326 & & \\
\hline \multicolumn{6}{|l|}{ Venues of sex with last male partner, P6M } \\
\hline Residence/bar/club/hotel & $3.3(36)$ & 1.0 & & & \\
\hline Bathhouse/sauna/park/public rest room & $6.6(9)$ & $2.1(1.0-4.4)$ & 0.0553 & & \\
\hline
\end{tabular}


TABLE 2: Continued.

\begin{tabular}{|c|c|c|c|c|c|}
\hline Variable & Recent infection \% $(N)$ & OR $(95 \% \mathrm{CI})$ & $P$ & $\operatorname{AOR}(95 \% \mathrm{CI})$ & $P$ \\
\hline \multicolumn{6}{|l|}{ Unprotected insertive anal sex with last male partner, P6M } \\
\hline No & $2.7(14)$ & 1.0 & & & \\
\hline Yes & $3.8(12)$ & $1.4(0.6-3.1)$ & 0.3894 & & \\
\hline \multicolumn{6}{|l|}{ Unprotected receptive anal sex with last male partner, P6M } \\
\hline No & $4.8(18)$ & 1.0 & & & \\
\hline Yes & $5.6(16)$ & $1.2(0.6-2.3)$ & 0.6490 & & \\
\hline \multicolumn{6}{|l|}{ Knew the HIV status of most recent male partner } \\
\hline Yes & $3.1(13)$ & 1.0 & & & \\
\hline No & $3.9(32)$ & $1.3(0.7-2.4)$ & 0.4914 & & \\
\hline \multicolumn{6}{|l|}{ Disclosed HIV status to most recent male partner } \\
\hline Yes & $3.4(21)$ & 1.0 & & & \\
\hline No & $4.0(24)$ & $1.2(0.7-2.2)$ & 0.5677 & & \\
\hline \multicolumn{6}{|l|}{ Bisexual } \\
\hline No & $3.1(32)$ & 1.0 & & 1.0 & \\
\hline Yes & $6.1(13)$ & $2.0(1.1-4.0)$ & 0.0339 & $2.1(1.1-4.1)$ & 0.0317 \\
\hline \multicolumn{6}{|l|}{ Ever had sex with a woman } \\
\hline Yes & $4.1(26)$ & 1.0 & & & \\
\hline No & $3.1(19)$ & $0.7(0.4-1.4)$ & 0.3445 & & \\
\hline \multicolumn{6}{|l|}{ Had female sexual partner, $\mathrm{P} 6 \mathrm{M}$} \\
\hline Yes & $3.1(6)$ & 1.0 & & & \\
\hline No & $3.7(39)$ & $1.2(0.5-2.8)$ & 0.7169 & & \\
\hline \multicolumn{6}{|l|}{ Unprotected sex with most recent female partner, P6M } \\
\hline No & $4.4(2)$ & 1.0 & & & \\
\hline Yes & $2.8(4)$ & $0.6(0.1-3.5)$ & 0.5990 & & \\
\hline \multicolumn{6}{|l|}{ Received a test for HIV, P12M } \\
\hline Yes & $3.4(24)$ & 1.0 & & & \\
\hline No & $3.9(21)$ & $1.2(0.6-2.1)$ & 0.5916 & & \\
\hline \multicolumn{6}{|l|}{ Received free condoms/lubricant, P12M } \\
\hline Yes & $3.4(33)$ & 1.0 & & & \\
\hline No & $4.5(12)$ & $1.4(0.7-2.7)$ & 0.3725 & & \\
\hline \multicolumn{6}{|l|}{ Bought condoms, P12M } \\
\hline Yes & $4.4(31)$ & 1.0 & & & \\
\hline No & $2.5(14)$ & $0.6(0.3-1.1)$ & 0.0768 & & \\
\hline \multicolumn{6}{|l|}{ Received free STD examination or treatment } \\
\hline Yes & $3.8(22)$ & 1.0 & & & \\
\hline No & $3.4(23)$ & $0.9(0.5-1.7)$ & 0.7632 & & \\
\hline \multicolumn{6}{|l|}{ Received free VCT } \\
\hline Yes & $3.9(25)$ & 1.0 & & & \\
\hline No & $3.2(20)$ & $0.8(0.4-1.5)$ & 0.4989 & & \\
\hline \multicolumn{6}{|l|}{ Main resource of knowledge of HIV/AIDS } \\
\hline Internet & $3.4(16)$ & 1.0 & & & \\
\hline $\begin{array}{l}\text { Friend/relative/sex partner/peer education/TV/newspapers/ } \\
\text { doctors/VCT/school education }\end{array}$ & $3.7(29)$ & $1.1(0.6-2.0)$ & 0.7657 & & \\
\hline \multicolumn{6}{|l|}{ Smoking, P12M } \\
\hline No & $3.7(28)$ & 1.0 & & & \\
\hline Yes & $3.5(17)$ & $0.9(0.5-1.7)$ & 0.8265 & & \\
\hline $\begin{array}{l}\text { Negative attitudes towards safe sex }(\text { Cronbach's alpha }=0.94, \\
\text { range }=15-60, \text { median }=20)\end{array}$ & na & $1.1(1.0-1.1)$ & 0.0006 & $1.1(1.0-1.1)$ & 0.0012 \\
\hline
\end{tabular}


TABLE 2: Continued.

\begin{tabular}{|c|c|c|c|c|}
\hline Variable & Recent infection \% $(N)$ & OR $(95 \%$ CI $)$ & $P$ & \multirow[t]{2}{*}{ AOR (95\% CI) } \\
\hline Current t & & & & \\
\hline No & $3.2(34)$ & 1.0 & & \\
\hline Yes & $6.0(11)$ & $1.9(1.0-3.9)$ & 0.0639 & \\
\hline \multicolumn{5}{|l|}{ Year } \\
\hline 2009 & $3.1(15)$ & 1.0 & & \\
\hline 2010 & $5.2(19)$ & $1.7(0.8-3.4)$ & 0.1351 & \\
\hline 2011 & $2.7(11)$ & $0.9(0.4-1.9)$ & 0.7018 & \\
\hline
\end{tabular}

* RMB: renminbi, approximately US \$0.15; P12M: in the past 12 months; P6M: in the past 6 months; OR: odds ratio; CI: confidence interval; AOR: adjusted odds ratio; NA: not applicable.

antiretroviral therapy or who have AIDS but with declining antibody levels, the BED assay may overestimate incidence rates $[20,44]$. Lastly, although $\mathrm{RDS}$ is effective in reaching hard-to-reach populations, it is based on a dual incentive regime; therefore, financial reward may attract more lowincome people to participate in the study which may limit the representativeness of the sample [30]. Although there are limitations in our study, it adds to our understanding of the dynamics of HIV infections among Beijing MSM.

This study, together with our previous study findings, demonstrated a disturbing rise of HIV infections among Beijing's MSM. HIV disproportionately affected MSM who were bisexual, short-term Beijing residents, with a negative attitude towards safe sex. These findings underscored the urgency of increasing effective and better-targeted intervention services to stop the rapid spread of the virus, which includes risk reduction education, condom promotion and distribution, encouraging early and frequent HIV testing, and expanding the coverage of HIV prevention measures. These interventions will help curb the spread of HIV infection inside and outside the MSM population.

\section{Conflict of Interests}

The authors declare that they have no conflict of interests.

\section{Authors' Contribution}

Xuefeng Li performed data retrieval and statistical analyses to prepare and write the initial draft of the paper. Yuhua Ruan and Yan Xiao were the PI of this study and constructed the conceptual framework of the work. Hongyan Lu, Yuejuan Zhao, Dongyan Xia, Yanming Sun, and Xiong He contributed to data collection and survey organization. Yujiang Jia, Catherine Cox, and Yiming Shao provided assistance in the conceptualization of the paper and contributed to revising and editing the final draft of the paper. All authors have read and approved the final version of the paper.

\section{Acknowledgments}

This study was supported by Grants from the National Natural Science Foundation of China (81273188), the National
Institutes of Health (nos. R01 AI078933 and 5D43TW00103513), the Ministry of Science and Technology of China (2012ZX10001-002), the International Development Research Center of Canada (Grant no. 104519-010), and Chinese State Key Laboratory for Infectious Disease Development Grant (2012SKLID103).

\section{References}

[1] L. Lu, M. Jia, Y. Ma et al., "The changing face of HIV in China," Nature, vol. 455, no. 7213, pp. 609-611, 2008.

[2] Y. Jia, F. Lu, X. Sun, and S. H. Vermund, "Sources of data for improved surveillance of HIV/AIDS in China," Southeast Asian Journal of Tropical Medicine and Public Health, vol. 38, no. 6, pp. 1041-1052, 2007.

[3] Z. Wu, K. Rou, and H. Cui, "The HIV/AIDS epidemic in China: history, current strategies and future challenges," AIDS Education and Prevention A, vol. 16, no. 3, pp. 7-17, 2004.

[4] Y. Jia, F. Lu, G. Zeng et al., "Predictors of HIV infection and prevalence for syphilis infection among injection drug users in China: community-based surveys along major drug trafficking routes," Harm Reduction Journal, vol. 5, no. 1, article 29, 2008.

[5] Y. Jia, J. Sun, L. Fan et al., "Estimates of HIV prevalence in a highly endemic area of China: Dehong Prefecture, Yunnan Province," International Journal of Epidemiology, vol. 37, no. 6, pp. 1287-1296, 2008.

[6] J. Huang, J. Jiang, J. Li et al., "Prevalence and correlates of sexual risk behaviors among drug users in western China: implications for HIV transmission," AIDS Research and Human Retroviruses, vol. 29, no. 4, pp. 673-680, 2013.

[7] State Council AIDS Working Committee Office and Un Theme Group on HIV/AIDS in China, "A Joint Assessment of HIV/ AIDS Prevention, Treatment and Care in China (2007)," Beijing 2008, http://www.docin.com/p-52176633.html.

[8] Y. Ruan, F. Luo, Y. Jia et al., "Risk factors for syphilis and prevalence of HIV, hepatitis $B$ and $C$ among men who have sex with men in Beijing, China: implications for HIV prevention," AIDS and Behavior, vol. 13, no. 4, pp. 663-670, 2009.

[9] Y. Ruan, D. Li, X. Li et al., "Relationship between syphilis and HIV infections among men who have sex with men in Beijing, China," Sexually Transmitted Diseases, vol. 34, no. 8, pp. 592$597,2007$.

[10] W. Cai, T. Feng, J. Tan et al., "A survey of the characteristics and STD/HIV infection of homosexuality in Shenzhen," China Journal of Preventive Medicine, vol. 32, pp. 328-330, 2005. 
[11] Q. He, Y. Wang, P. Lin et al., "Potential bridges for HIV infection to men who have sex with men in Guangzhou, China," AIDS and Behavior, vol. 10, no. 4, supplement, pp. S17-S23, 2006.

[12] K. Choi, Z. Ning, S. E. Gregorich, and Q. Pan, “The influence of social and sexual networks in the spread of HIV and syphilis among men who have sex with men in Shanghai, China," Journal of Acquired Immune Deficiency Syndromes, vol. 45, no. 1, pp. 77-84, 2007.

[13] J. Jiang, N. Cao, J. Zhang et al., "High prevalence of sexually transmitted diseases among men who have sex with men in Jiangsu Province, China," Sexually Transmitted Diseases, vol. 33, no. 2, pp. 118-123, 2006.

[14] S. Ruan, H. Yang, Y. Zhu et al., "HIV prevalence and correlates of unprotected anal intercourse among men who have sex with men, Jinan, China," AIDS and Behavior, vol. 12, no. 3, pp. 469475, 2008.

[15] Y. Ruan, X. Cao, H. Qian et al., "Syphilis among female sex workers in southwestern China: potential for HIV transmission," Sexually Transmitted Diseases, vol. 33, no. 12, pp. 719-723, 2006.

[16] Y. Ruan, Y. Jia, X. Zhang et al., "Incidence of HIV-1, syphilis, hepatitis $B$, and hepatitis $c$ virus infections and predictors associated with retention in a 12-month follow-up study among men who have sex with men in Beijing, China," Journal of Acquired Immune Deficiency Syndromes, vol. 52, no. 5, pp. 604610, 2009.

[17] Y. Xiao, X. Ding, C. Li, J. Liu, J. Sun, and Y. Jia, "Prevalence and correlates of hiv and syphilis infections among men who have sex with men in chongqing municipality, China," Sexually Transmitted Diseases, vol. 36, no. 10, pp. 647-656, 2009.

[18] E. P. F. Chow, D. P. Wilson, J. Zhang, J. Jing, and L. Zhang, "Human immunodeficiency virus prevalence is increasing among men who have sex with men in China: findings from a review and meta-analysis," Sexually Transmitted Diseases, vol. 38, no. 9, pp. 845-857, 2011.

[19] L. Wang, J. L. Norris, D. M. Li, W. Guo, Z. W. Ding, and N. Wang, "HIV prevalence and influencing factors analysis of sentinel surveillance among men who have sex with men in China, 2003-2011," Chinese Medical Journal, vol. 125, no. 11, pp. 18571861, 2012.

[20] B. S. Parekh and J. S. McDougal, "Application of laboratory methods for estimation of HIV-1 incidence," Indian Journal of Medical Research, vol. 121, no. 4, pp. 510-518, 2005.

[21] A. A. Kim, T. Hallett, J. Stover et al., "Estimating HIV incidence among adults in Kenya and Uganda: a systematic comparison of multiple methods," PLoS ONE, vol. 6, no. 3, Article ID e17535, 2011.

[22] T. B. Hallett, B. Zaba, J. Todd et al., "Estimating incidence from prevalence in generalised HIV epidemics: methods and validation," PLoS Medicine, vol. 5, no. 4, article e80, 2008.

[23] Y. T. Duong, M. Qiu, A. K. De et al., "Detection of recent HIV-1 infection using a new limiting-antigen avidity assay: potential for HIV-1 incidence estimates and avidity maturation studies," PLoS ONE, vol. 7, no. 3, Article ID e33328, 2012.

[24] T. Bärnighausen, C. Wallrauch, A. Welte et al., "HIV incidence in rural South Africa: comparison of estimates from longitudinal surveillance and cross-sectional cBED assay testing," PLoS ONE, vol. 3, no. 11, Article ID e3640, 2008.

[25] C. Serna-Bolea, N. de Deus, S. Acácio et al., "Recent HIV-1 infection: identification of individuals with high viral load setpoint in a voluntary counselling and testing centre in rural mozambique," PLoS ONE, vol. 7, no. 2, Article ID e31859, 2012.
[26] O. Laeyendecker, M. Kulich, D. Donnell et al., "Development of methods for cross-sectional HIV incidence estimation in a large, community randomized trial," PLoS ONE, vol. 8, no. 11, Article ID e78818, 2013.

[27] X. Li, H. Lu, X. Ma et al., "HIV/AIDS-related stigmatizing and discriminatory attitudes and recent HIV testing among men who have sex with men in Beijing," AIDS and Behavior, vol. 16, no. 3, pp. 499-507, 2012.

[28] X. Li, H. Lu, H. F. Raymond et al., "Untested and undiagnosed: barriers to HIV testing among men who have sex with men, Beijing, China," Sexually Transmitted Infections, vol. 88, no. 3, pp. 187-193, 2012.

[29] B. S. Parekh, M. S. Kennedy, T. Dobbs et al., "Quantitative detection of increasing HIV type 1 antibodies after seroconversion: a simple assay for detecting recent HIV infection and estimating incidence," AIDS Research and Human Retroviruses, vol. 18, no. 4, pp. 295-307, 2002.

[30] L. G. Johnston, M. Malekinejad, C. Kendall, I. M. Iuppa, and G. W. Rutherford, "Implementation challenges to using respondent-driven sampling methodology for HIV biological and behavioral surveillance: field experiences in international settings," AIDS and Behavior, vol. 12, no. 4, supplement, pp. S131S141, 2008.

[31] X. Ma, Q. Zhang, X. He et al., "Trends in prevalence of HIV, syphilis, hepatitis $C$, hepatitis $B$, and sexual risk behavior among men who have sex with men: results of 3 consecutive respondent-driven sampling surveys in Beijing, 2004 through 2006," Journal of Acquired Immune Deficiency Syndromes, vol. 45, no. 5, pp. 581-587, 2007.

[32] S. Li, X. Zhang, X. Li et al., "Detection of recent HIV-1 infections among men who have sex with men in Beijing during 20052006," Chinese Medical Journal, vol. 121, no. 12, pp. 1105-1108, 2008.

[33] M. Han, L. Feng, Y. Jiang et al., "Surveillance on HIV-1 incidence among men who have sex with men in Chongqing, China, 2006-2008," Chinese Journal of Epidemiology, vol. 30, no. 9, pp. 878-881, 2009.

[34] M. Han, L. G. Feng, J. K. Zhao et al., "A survey of BED HIV-1 incidence among MSM in Chongqing of China, 2009," Chinese Journal of AIDS \& STD, vol. 6, pp. 654-656, 2011.

[35] H. Y. Hu, S. Shen, X. P. Huan, H. J. Yan, Y. Xiao, and Y. Jiang, "Estimation of HIV-1 ncidence of MSM in Jiangsu Province with BED-CEIA assay," Acta Universitatis Medicinalis Nanjing, vol. 30, pp. 467-471, 2010.

[36] K. A. Fenton and C. M. Lowndes, "Recent trends in the epidemiology of sexually transmitted infections in the European Union," Sexually Transmitted Infections, vol. 80, no. 4, pp. 255-263, 2004.

[37] T. A. Peterman, J. D. Heffelfinger, E. B. Swint, and S. L. Groseclose, "The changing epidemiology of syphilis," Sexually Transmitted Diseases, vol. 32, no. 10, supplement, pp. S4-S10, 2005.

[38] K. Yun, J. J. Xu, K. H. Reilly et al., "Prevalence of bisexual behaviour among bridge population of men who have sex with men in China: a meta-analysis of observational studies," Sexually Transmitted Infections, vol. 87, no. 7, pp. 563-570, 2011.

[39] E. P. F. Chow, D. P. Wilson, and L. Zhang, "Original article for BMC Infectious Diseases What is the potential for bisexual men in China to act as a bridge of HIV transmission to the female population? Behavioural evidence from a systematic review and meta-analysis," BMC Infectious Diseases, vol. 11, article 242, 2011.

[40] Y. Feng, Z. Wu, and R. Detels, "Evolution of men who have sex with men community and experienced stigma among men who 
have sex with men in Chengdu, China," Journal of Acquired Immune Deficiency Syndromes, vol. 53, supplement 1, pp. S98-S103, 2010.

[41] J. X. Liu and K. Choi, "Experiences of social discrimination among men who have sex with men in Shanghai, China," AIDS and Behavior, vol. 10, no. 4, supplement, pp. S25-S33, 2006.

[42] H. Yang, C. Hao, X. Huan et al., "HIV incidence and associated factors in a cohort of men who have sex with men in Nanjing, China," Sexually Transmitted Diseases, vol. 37, no. 4, pp. 208-213, 2010.

[43] H. Thiede, R. A. Jenkins, J. W. Carey et al., "Determinants of recent HIV infection among Seattle-area men who have sex with men," American Journal of Public Health, vol. 99, supplement 1, pp. S157-S164, 2009.

[44] R. Brookmeyer, "Measuring the HIV/AIDS epidemic: approaches and challenges," Epidemiologic Reviews, vol. 32, no. 1, pp. 26-37, 2010. 


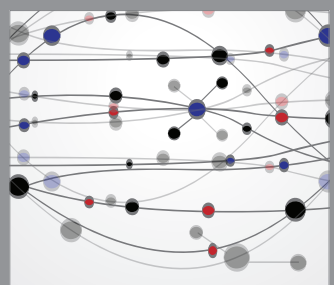

The Scientific World Journal
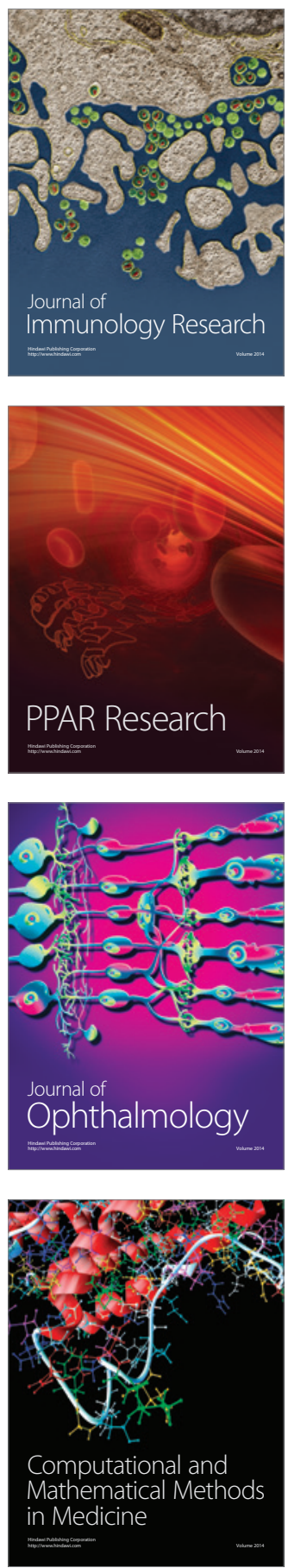

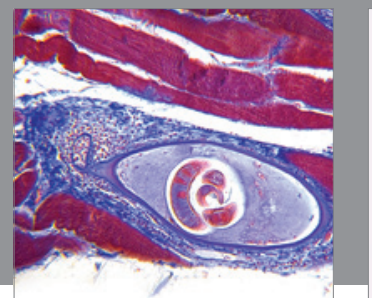

Gastroenterology

Research and Practice
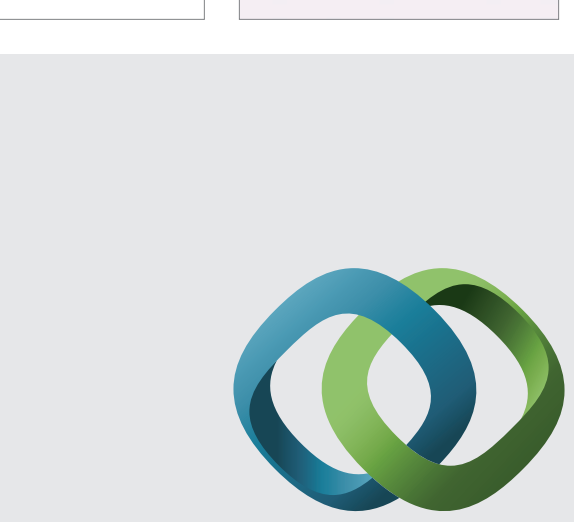

\section{Hindawi}

Submit your manuscripts at

http://www.hindawi.com
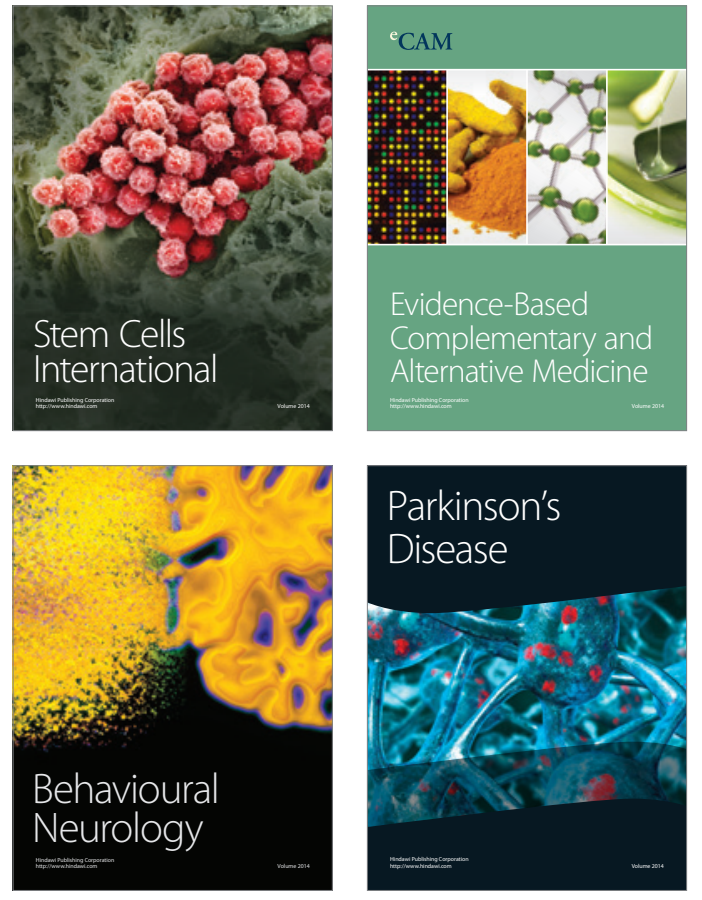
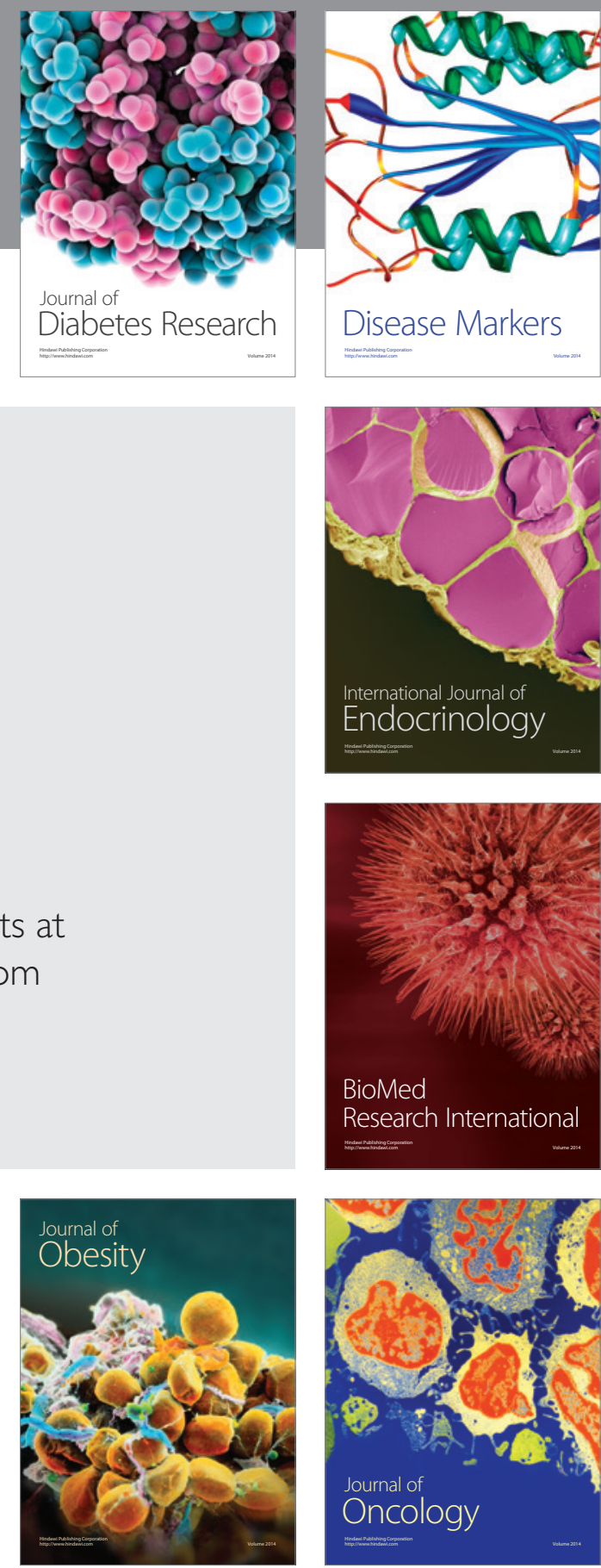

Disease Markers
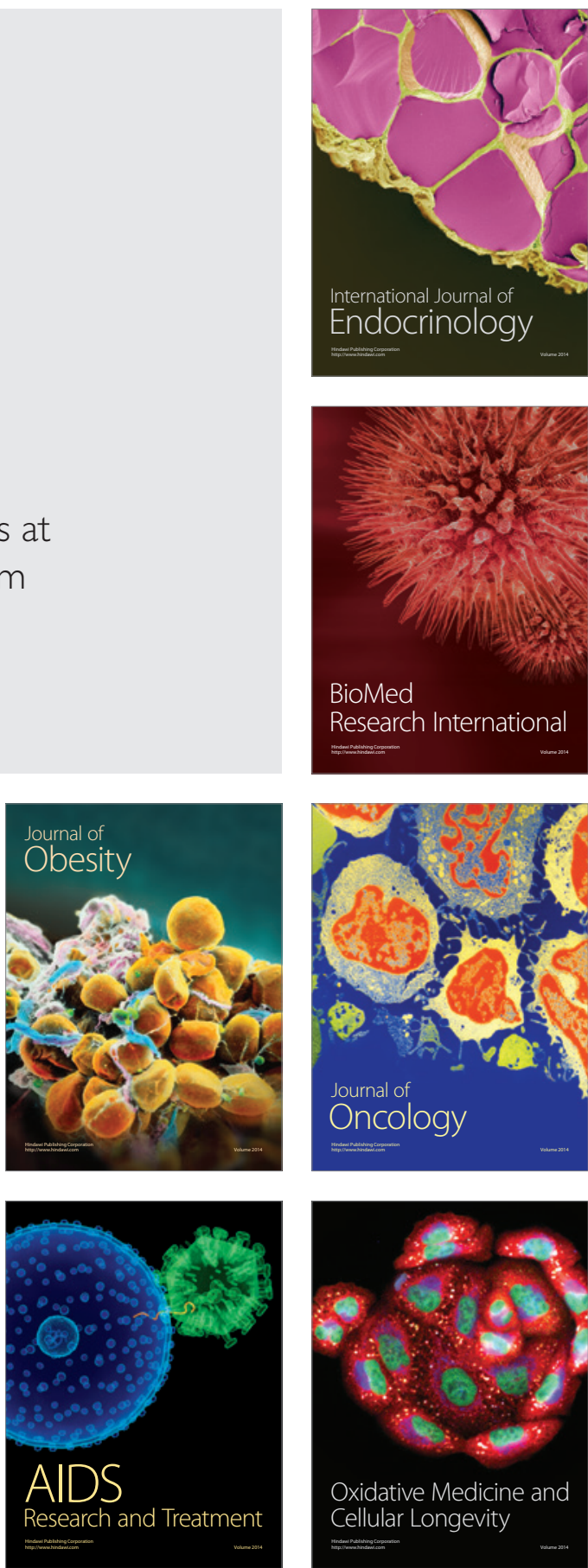